\title{
Assessment of White Spaces Quality in Rural Areas: a large-scale spectrum survey
}

\author{
$1^{\text {st }}$ Rodney Martinez Alonso \\ Information Technologies \\ Ghent University \\ Ghent, Belgium \\ rodney.martinezalonso@ugent.be
}

\author{
$2^{\text {nd }}$ Arley Coto Guerra \\ Research and Development \\ LACETEL \\ Havana, Cuba \\ arley@lacetel.cu
}

\author{
$6^{\text {th }}$ Luc Martens \\ Information Technologies \\ Ghent University \\ Ghent, Belgium \\ luc1.martens@ugent.be
}

\author{
$3^{\text {rd }}$ Ernesto Fontes Pupo \\ Research and Development \\ LACETEL \\ Havana, Cuba \\ fontes@lacetel.cu
}

\author{
$4^{\text {th }}$ David Plets \\ Information Technologies \\ Ghent University \\ Ghent, Belgium \\ david.plets@ugent.be
}

\author{
Research and Development
Revillen Nieto \\ LACETEL \\ Havana, Cuba \\ glauco@lacetel.cu
}

\begin{abstract}
Spectrum is a valuable resource for broadcasting and wireless communications. Despite the fact that most of the sub-1-GHz spectrum is granted, spectrum surveys in suburban areas reveal a poor usage efficiency (less than $20 \%$ on average). Cognitive Radio for 5G next generation radio is a potential technology for dynamically exploit the spectrum white spaces, particularly in low populated rural areas. However, not all the empty spectrum is useful for providing broadband wireless connectivity guaranteeing a certain Quality of Service $(Q \circ S)$ or without causing harmful interference to the primary licensed services. In this paper we perform a large scale-spectrum survey and measurement campaign in two rural areas. We also quantify the white spaces and its real potential for secondary services. Our research reveals that, on average, less than $11 \%$ of the spectrum is in use in the surveyed rural areas. However, in one area only $60 \%$ of the white spaces can be used for a commercial service. QoS

Index Terms-Spectrum Utilization, Spectrum Survey, TVWS,
\end{abstract}

\section{INTRODUCTION}

The radio spectrum is a valuable and limited resource. Increasing spectral efficiency has been a key target, particularly important for broadcasting and wireless communication technologies. However, for a single channel with a certain bandwidth and noise characteristic, there is a maximum data rate for which data can be sent without errors (Shannon capacity limit). Several investigations have been conducted for approaching wireless technologies to the theoretical maximum limit. The innovation of low-density parity check codes (LDPC) improved the capacity results achieved by turbo codes [1]. In [2] authors approached theoretically the Shannon limit by $0.0045 \mathrm{~dB}$. Nevertheless, for practical implementations a trade-off to the decoder hardware complexity and its power consumption should be considered. A real application of LDPC by the ATSC 3.0 digital television standard contributed

This paper has been supported in part by a grant from the Special Research Funds allocated by the Flemish Government to Ghent University, by LACETEL, and Rhode \& Schwarz, Belgium. to approach the Shannon limit by $1.3 \mathrm{~dB}$ to $1.8 \mathrm{~dB}$ [3]. LongTerm Evolution (LTE) implementations reported in [4] for mobile communications are not better than ATSC 3.0. The physical layer for $5 \mathrm{G}$ new generation radio is expected to be around $1 \mathrm{~dB}$ from the Shannon limit [5].

The margin for improvement of spectral efficiency with respect to the Shannon limit is small. Although, some initial research suggest that this limit could be over exceeded by quantic technologies, these theoretical approaches cannot be implemented with state-of-the-art technologies [6]. As such, it is not implemented for current or foreseen deployments of $5 \mathrm{G}$ new generation radio [5].

There is not a wide margin for improving technologies spectral efficiency respect to the Shannon limit. However, several spectrum surveys demonstrate that the spectrum usage in large cities and sub-urban areas is lower than 20\% [7], [8]. This means that the usage of the spectrum is highly inefficient, providing the potential for spectrum optimization by cognitiveradio-based technologies.

Studies have analyzed the Ultra-High Frequency (UHF) band white space availability in different areas [9]-[11]. However, the spectrum surveys, and considered metrics do not take into account the temporal availability of the spectrum i.e., temporal variations in the channel due to fading or even service demand. A channel not in use by the primary licensed services (white spaces) does not necessarily meet the requirements for guaranteeing an acceptable QoS for the wireless communication service or for avoiding harmful interference to the primary service. For instance, in rural areas the spectrum should be available more than $95 \%$ of the time.

In this paper we quantify the spectrum usage and the spectrum utility, i.e., the percentage of white spaces useful for providing secondary wireless services guaranteeing the required QoS and without causing harmful interference to the broadcasting services in rural scenarios. We perform a large-scale spectrum survey in two rural areas, consisting of 
experimental spectrum measurements and monitoring in the Sub-1 GHz bands. Here, we take into account the spatial and temporal availability of the spectrum. We propose two metrics for assessing the spectrum usage and spectrum utility from the collected data. In addition, we quantify the potential effectiveness of cognitive radio technologies for a higher spectrum usage efficiency by wireless telecommunications technologies for broadband connectivity in rural areas, and without causing harmful interference to the primary licensed services.

\section{METHOD}

\section{A. Measurement Scenarios}

For the spectrum survey, we considered two relatively low populated areas of Cuba and Belgium. As the subscriber (user) density decreases, the CAPEX and OPEX increase. As consequence the Average Revenue Per Subscriber (ARPU) is low, which discourage operators from investing in telecommunication infrastructure in these regions. Hence, low populated areas might benefit from utilizing unexploited spectrum in the lower frequency bands (e.g., the UHF band) [12]. In Cuba, the survey was carried out in Bejucal-Quivican. This is a mixed suburban-rural area of $169 \mathrm{~km}^{2}$. The population density in this region is approximately 120 people per $\mathrm{km}^{2}$. In Belgium, the survey was performed in Nevele, with an area of $52 \mathrm{~km}^{2}$. This is a Flemish town with a population density of 216 people per $\mathrm{km}^{2}$. Both environments have a mostly flat topology, comprising row houses and detached houses surrounded by large flat areas dedicated to farming.

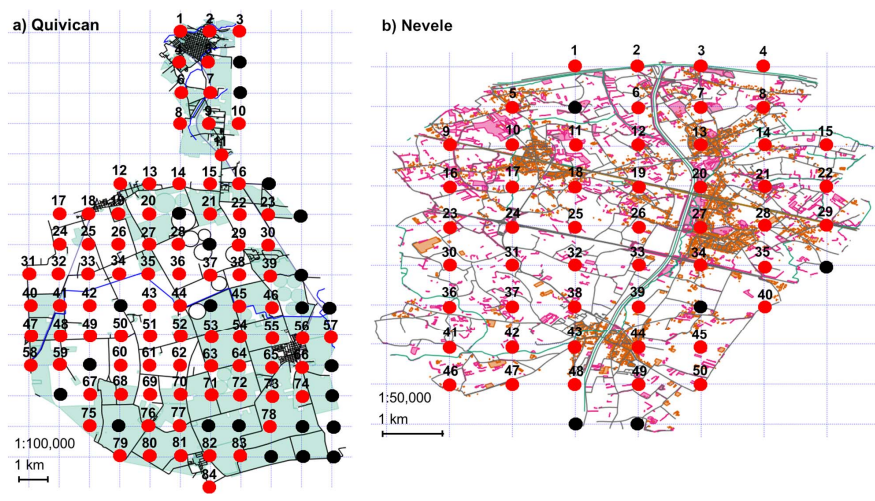

Fig. 1. Field trial area for the spectrum surveys: a) Bejucal-Quivican, Cuba b) Nevele, Belgium. Red dots: measurement locations; black dots locations where measurement is not feasible.

For choosing the measurement locations, a grid of $1 \mathrm{~km}$ by $1 \mathrm{~km}$ is created. The closest accessible site nearby the grid point location is chosen for a measurement, taking into account that some grid points were in private terrain or inaccessible by road. For the same reason it was not possible to find a suitable measurement location for 22 grid points in Bejucal-Quivican (black dots in Fig. 1a) and 5 locations in Nevele (black dots in Fig. 1b). In total, 84 locations have been investigated in Bejucal-Quivican (red dots in Fig. 1a) and 50 in Nevele (red dots in Fig. 1b).

\section{B. Measurement Setup}

Fig. 2 shows the measurement setup for surveying the signal level in the sub- $1 \mathrm{GHz}$ spectrum.

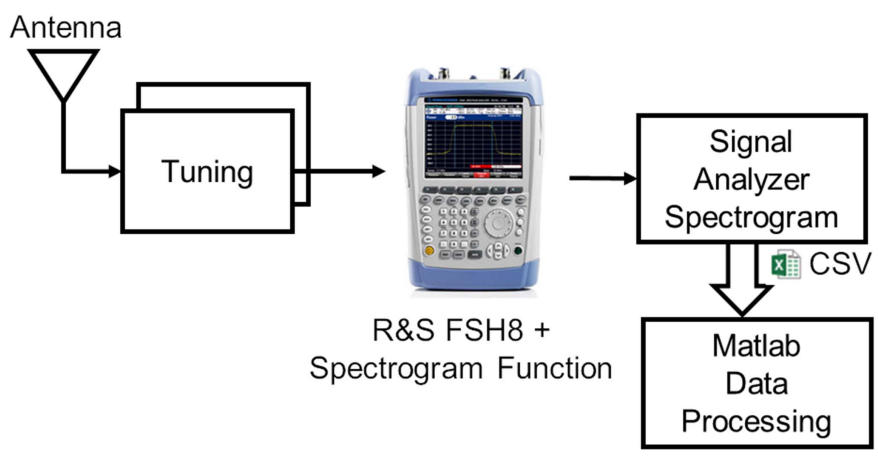

Fig. 2. Measurement setup for the spectrum survey

The signal level measurements are based on energy detection, receiving the signal by an omnidirectional antenna (Fig. 2). The antenna gain just compensates for the transmission line losses. The level at the input of the measurement device is equivalent to the signal level in the antenna input with an error of $\sim 2 \mathrm{~dB}$ in the measurement range. A proper correction according to the antenna and cables characteristics is performed. Here, we do not account for cross polarization effect. Notice that this effect will cause an additional protection margin between the primary and the secondary service in some use cases (cross polarization) or not effect at all (matching polarization). Approximately a $3 \mathrm{~dB}$ lose is expected between circular and vertical polarization and $20 \mathrm{~dB}$ loss (theoretically) between vertical and horizontal polarization. However, because there is no direct line of sight between the transmitter and the receiver, and because of the signal reflections in the real environment the maximum crosspolarization loss was lower than $3 \mathrm{~dB}$ (according to in-site measurements).

In the range of $170 \mathrm{MHz}$ to $1 \mathrm{GHz}$, the signals are tuned at each frequency with a frequency resolution of $\sim 1.31 \mathrm{MHz}$. We continuously scan the spectrum starting at $170 \mathrm{MHz}$ (instead of DC). This is because a key goal of the measurement campaign is to quantify the spectrum utility for secondary services in a rural area and the users' antenna dimensions for a commercial wireless service in the sub-170 $\mathrm{MHz}$ is not suitable for most applications.

The spectrum analyzer (Fig. 2) is set for performing up to 410 measurements at each frequency and we account for signal level variations due to fading effects. The measurements were performed in all locations at the peak spectrum utilization time at late morning (10:00 to 12:00) and afternoon (14:00 to 17:00) [13]. We monitored each location for a total time of $\sim 30$ minutes.

\section{Data processing and Metrics}

The CSV file for each location consists of a matrix of 631 frequencies (due to the frequency resolution) by 410 level 
measurements (due to the sampling time). For each frequency and location, we determine the median and the $95^{\text {th }}$-percentile of the signal level. The median level in the time domain is equivalent to a $50 \%$ duty cycle, i.e., the percentage of time the signal level does not exceed a certain threshold. Each frequency is independently evaluated. Equation 1 defines the metric to account for the spectrum utilization SU [\%] at each location. This metric defines if a service is using a particular segment of the spectrum at any given location.

$S U=\frac{100 \cdot \sum_{i=0}^{630} r \cdot k_{r \cdot i+170}}{830}\left\{\begin{array}{lll}k=1 & \text { if } & L_{50} \geq-95 \mathrm{dBm} \\ k=0 & \text { if } & L_{50}<-95 \mathrm{dBm}\end{array}\right.$

where $i$ is the frequency index, $r$ is the frequency resolution considered for the measurements $(\sim 1.31 \mathrm{MHz}), k$ is a unitary value that defines whether this portion of the spectrum is occupied or not, according to a certain criterion for the median signal level $L_{50}$ throughout time. Here, we consider $-95 \mathrm{dBm}$ as the utilization threshold. We consider the signal level threshold based on the protection contour of the broadcast transmitters recommended in [9]. Hence, the spectrum utilization SU represents the total bandwidth for which a median signal level higher than $-95 \mathrm{dBm}$ is recorded. Finally, this value can be normalized by dividing for the total assigned spectrum by the regulatory authorities below $1 \mathrm{GHz}$.

Not all the spectrum that is un-utilized can be used for deploying another wireless communication service. The primary service in all cases needs to be protected from harmful interference [13]. Also, a minimum temporal availability of the spectrum should be granted for guaranteeing the required quality of service for the secondary wireless service. The temporal availability required for wireless services is in the range of $95 \%$ to more than $99 \%$, depending on the density of users and the trade-off with the network cost. The TVWS utility $U$ is defined considering the $95^{t h}$-percentile of the signal level $L_{95}$ for the threshold of $-95 \mathrm{dBm}$ [9]. The $U$ [\%] is determined as follows:

$$
U=\frac{100 \cdot \sum_{i=0}^{630} r \cdot k_{r \cdot i+170}}{830}\left\{\begin{array}{lll}
k=1 & \text { if } & L_{95}<-95 d B m \\
k=0 & \text { if } & L_{95} \geq-95 d B m
\end{array}\right.
$$

\section{RESULTS}

Fig. 3 shows the observed signal level as a function of frequency for a $50 \%$ duty cycle considering the median of all locations $\left(L_{50}\right)$ and the values for which the signal level is not exceeded in $95 \%$ of the locations $\left(L_{95}\right)$. In the BejucalQuivican region, the band section with the highest median level (solid black line in Fig. 3), corresponds to the downlink from $3 \mathrm{G}$ base stations $(\sim 950 \mathrm{MHz})$ providing mobile communication services (approximately $-82 \mathrm{dBm}$ accounting for all locations and time samples). In Nevele, also the highest median (solid black line in Fig. 3) corresponds to mobile services (LTE-A) in the $800 \mathrm{MHz}$ band (approximately $-80 \mathrm{dBm})$.

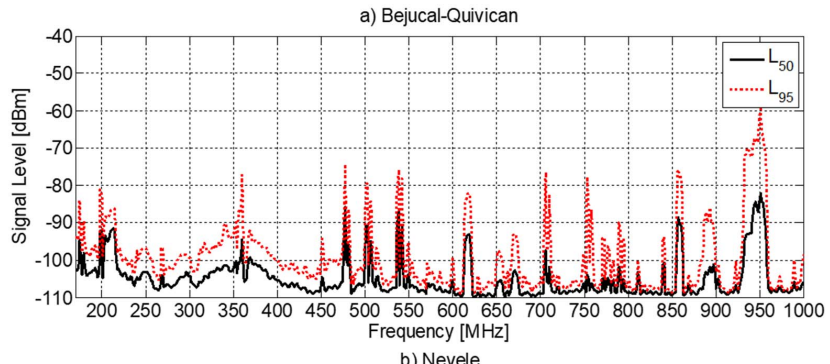

b) Nevele

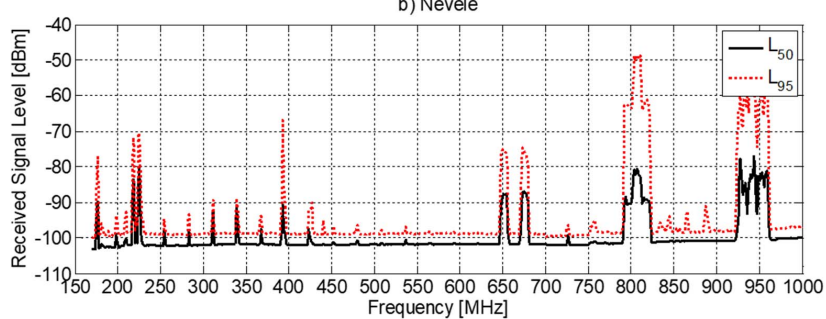

Fig. 3. Received signal level for a) Bejucal-Quivican and b) Nevele, considering all measurements in the spatial and temporal domain. $L_{50}$ is the median signal level (straight black line) and $L_{95}$ the $95^{t h}$-percentile of the signal level (dotted red line).

A typical characteristic of the rural area is the low population density. As a consequence, the uplink power level $(832 \mathrm{MHz}-862 \mathrm{MHz})$ is considerably small when averaged over time and space domains. The highest spectral emission in Bejucal-Quivican corresponds to the Global System for Mobile Communications (GSM) service in the $900 \mathrm{MHz}$ band with a level of $-60 \mathrm{dBm}$. In Bejucal-Quivican, the second largest spectrum emission is from broadcasting services at UHF band IV and V. There are 11 television channels in use, with a received channel power above $-95 \mathrm{dBm}$ for the $95^{t h}$ percentile (dotted red line in Fig. 3). Some emissions are not generated in the area of the survey but from nearby locations. In Nevele, the highest spectral emission for the $95^{t h}$-percentile corresponds also to mobile services (LTE-A) in the $800 \mathrm{MHz}$ band with a received signal level near $-50 \mathrm{dBm}$. The emission footprints in Nevele are lower than in Quivican-Bejucal, with most of the UHF band not in use by any service.

Fig. 4 shows a map with the percentage of spectrum utilization from the measurement campaign data and quantified by means of Equation 1. The average Spectrum Utilization for the defined criterion (see section II-C) is lower than 7.6\% in Bejucal-Quivican area (Fig. 4a) and lower than $11.0 \%$ in Nevele (Fig. 4b). These values are considerably lower than the reported spectrum utilization for dense urban and suburban areas (approximately $20 \%$ on average) [7], [8]. In Nevele the spectrum utilization is quite uniform and never higher than 13\% (in Fig. 4b).

At a small number of locations in Bejucal-Quivican (2 of 84 ), the spectrum utilization is slightly higher than $20 \%$ (in Fig. 4a). This is mainly because these locations in BejucalQuivican are closer to suburban areas with a higher signal emission density, i.e., higher contributions from mobile services uplink, aeronautical services, railway communications, and air traffic control. 


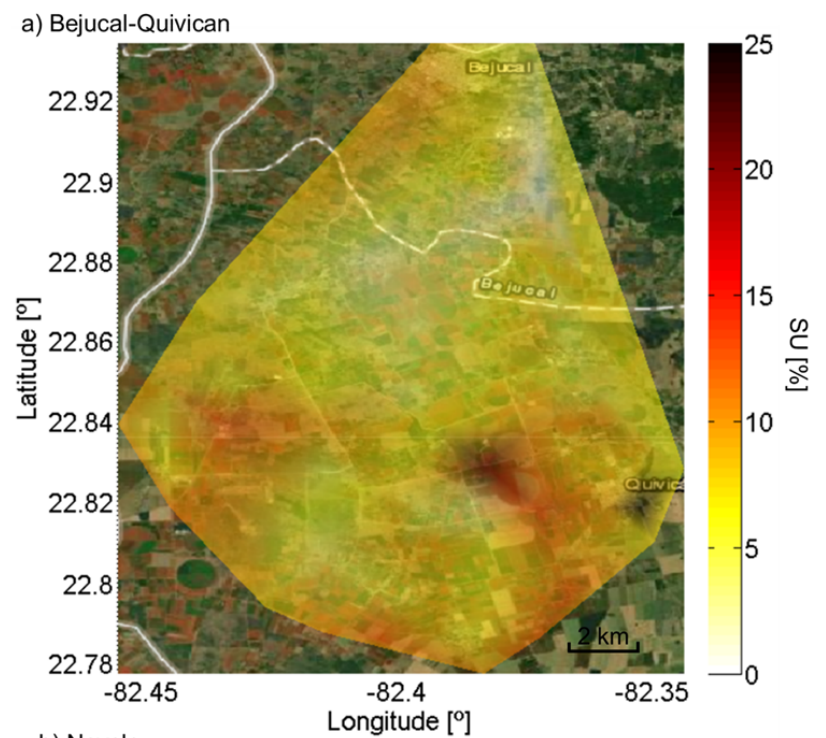

b) Nevele

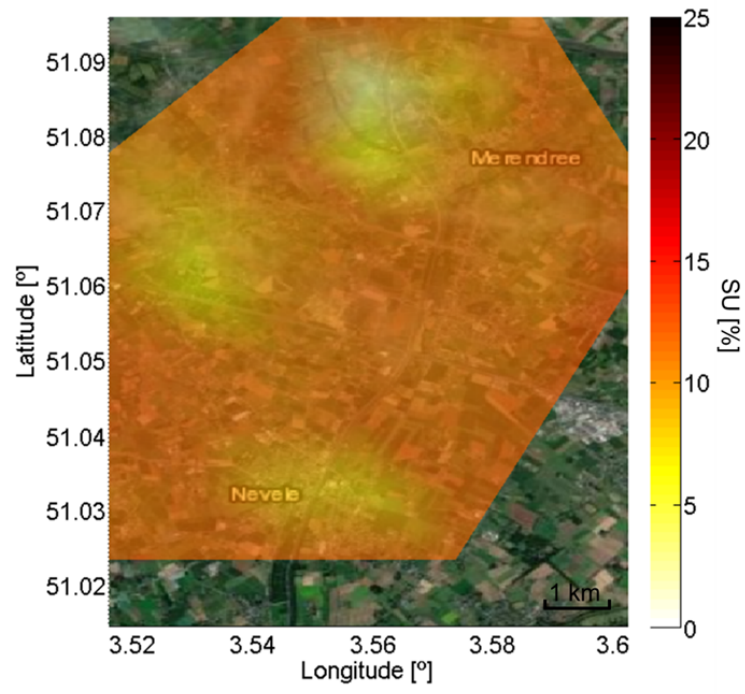

Fig. 4. Spectrum Utilization (SU) for the different measurement locations in a) Bejucal-Quivican, Cuba and b) Nevele, Belgium

No all the remaining spectrum, not in use by communication services, can be useful for secondary services due to temporal availability requirements. Fig. 5 shows the spectrum utility considering the data from the measurement campaign and calculated by means of Equation (2).

For both Bejucal-Quivican and Nevele, the average white space utility is $87 \%$. This means that $87 \%$ of the spectrum can be reused by secondary services based on a temporal availability of $95 \%$. In Bejucal-Quivican the white spaces are not uniformly distributed. For a small number of locations, the spectrum utility is lower than $60 \%$. This is because of the differences in propagation conditions and the density of services using the spectrum. Some locations closer to the suburban area and a cargo airport have a higher utilization of amateur radio, and aeronautic navigation. In Nevele, there is a more uniform distribution of white spaces in the area with a) Bejucal-Quivican
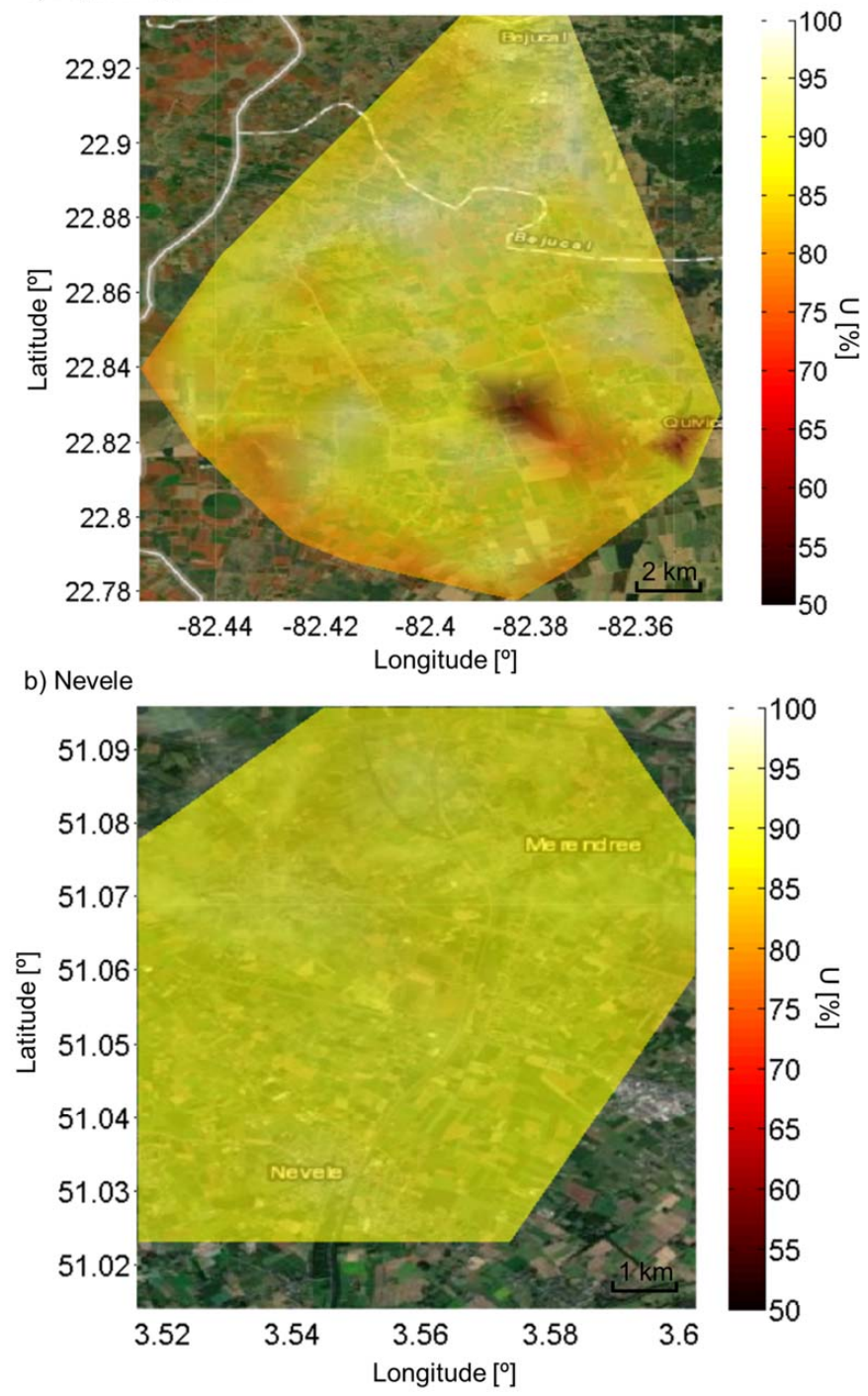

Fig. 5. White Space Utility (U) for the different measurement locations: a) Bejucal-Quivican and b) Nevele.

a standard deviation of the White Space Utility lower than 3\%.

In locations with a considerably lower White Space Utility compared to nearby locations the interference to broadcasting services might be considerably higher. Fig. 6 shows the spectrogram of the worst location compared to the best location in terms of spectrum availability.

In the worst locations the variability of channels availability throughout time is higher. For critical propagation conditions and a protection contour of the transmitter lower than $20 \mathrm{~km} \mathrm{[9],} \mathrm{[14],} \mathrm{the} \mathrm{interference} \mathrm{threshold} \mathrm{of}-95 \mathrm{dBm}$ might not be enough for avoiding harmful interference to the broadcasting services. In an experimental assessment of the harmful interference caused by a cognitive radio network with distributed architecture to a broadcasting service for critical conditions the percentage of video degradation was higher than $60 \%$ [14]. 


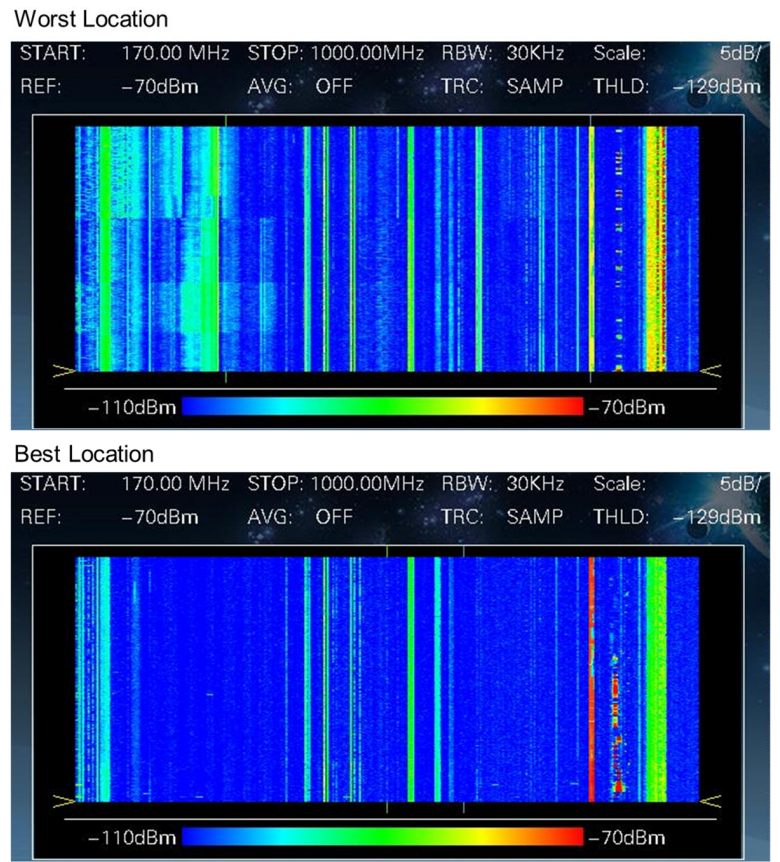

Fig. 6. Spectrogram of selected locations

\section{CONCLUSiOnS}

The spectrum efficiency in two typical rural areas was considerably lower than those reported by previous surveys for sub-urban and urban areas. On average less than $11 \%$ of the spectrum is in use in the surveyed rural areas. Nevertheless, no all the remaining spectrum can be used for providing wireless connectivity with an acceptable QoS. The potentially useful white spaces are on average $87 \%$ of the assigned spectrum. More than $680 \mathrm{MHz}$ could be available for 5G new generation radio by using cognitive radio; taking into account that this technique allows dynamic access of the spectrum. Nevertheless, spectrum partitioning, and irregular white space availability could be a challenge in some rural areas. In the particular case of Bejucal-Quivican, the spectrum is highly fragmented and not uniformly distributed in the area, making difficult for a long-term solution with traditional cognitive radio technologies based on distributed spectrum management. Without a centralized architecture capable of minimizing the interference of the hidden nodes, the cognitive radio will potentially cause harmful interference to the primary licensed services in some locations of this surveyed area.

\section{ACKNOWLEDGMENT}

We would like to thanks the administrative and logistic arrangements made by Leen Verloock, Karien Hemelsoen and Isabelle Van der Elstraeten. We also would like to thanks to the local authorities of Nevele and Deinze in Belgium as well as Bejucal and Quivican in Cuba.

\section{REFERENCES}

[1] T. J. Richardson and R. L. Urbanke, "The Capacity of Low-Density Parity-Check Codes Under Message-Passing Decoding," IEEE TRANS-
ACTIONS ON INFORMATION THEORY, vol. 47, no. 2, pp. 599-618, 2001.

[2] S.-Y. Chung, G. D. J. Forney, T. J. Richardson and R. Urbanke, "On the design of low-density parity-check codes within $0.0045 \mathrm{~dB}$ of the Shannon limit," IEEE COMMUNICATIONS LETTERS, vol. 5, no. 2, pp. 58-60, 2001.

[3] L. Fay, L. Michael, D. Gómez-Barquero, N. Ammar and M. W. Caldwell, "An Overview of the ATSC 3.0 Physical Layer Specification," IEEE TRANSACTIONS ON BROADCASTING, vol. 62, no. 1, pp. 159-171, 2016.

[4] M. Kalil, A. Shami and A. Al-Dweik, "QoS-Aware Power-Efficient Scheduler for LTE," IEEE TRANSACTIONS ON MOBILE COMPUTING, vol. 14, no. 8, pp. 1672-1685, 2015.

[5] P. Banelli, S. Buzzi, G. Colavolpe, A. Modenini, F. Rusek and A. Ugolini, "Modulation Formats and Waveforms for 5G Networks: Who Will Be the Heir of OFDM?," IEEE SIGNAL PROCESSING MAGAZINE, vol. 31, no. 6, pp. 80-93, 2014.

[6] L. Gyongyosi, S. Imre and H. V. Nguyen, "A Survey on Quantum Channel Capacities," IEEE Communications Surveys and Tutorials, vol. 20, no. 2, pp. 1149 - 1205, 2018.

[7] Shared Spectrum Company, "General Survey of Radio Frequency Bands - $30 \mathrm{MHz}$ to $3 \mathrm{GHz}$," Vienna, Virginia, 2010.

[8] V. Valenta, R. Marsalek, G. Baudoin and M. Villegas, "Survey on Spectrum Utilization in Europe: Measurements, Analyses and Observations," 5th International ICST Conference on Cognitive Radio Oriented Wireless Networks and Communications, 2010.

[9] J. van de Beek, J. Riihijarvi, A. Achtzehn and P. Mahonen, "TV White Space in Europe," IEEE TRANSACTIONS ON MOBILE COMPUTING, vol. 11, no. 2, pp. 178-188, 2012.

[10] A. Arteaga and A. Navarro, "Availability of TV White Spaces Using Spectrum Occupancy Information and Coverage Maps," IEEE LATIN AMERICA TRANSACTIONS, vol. 14, no. 6, pp. 2588-2591, 2016.

[11] P. Palka, "TV white space utility in urban and rural environments," in IEEE International Symposium on Broadband Multimedia Systems and Broadcasting, Ghent, Belgium, 2015.

[12] R. Stewart, D. Crawford, and A. Stirling. "TV White Space Communications and Networks," Woohead Publishing-Elsevier, 2018.

[13] R. Martinez Alonso, D. Plets, E. Fontes Pupo, M. Deruyck, L. Martens, G. Guillen Nieto and W. Joseph, "IoT-Based Management Platform for Real-Time Spectrum and Energy Optimization of Broadcasting Networks," Wireless Communications and Mobile Computing, vol. 2018, pp. 1-14, 2018.

[14] R. Martinez Alonso, D. Plets, M. Deruyck, L. Martens, G. Guillen Nieto, and W. Joseph, "Dynamic Interference Optimization in Cognitive Radio Networks for Rural and Suburban Areas," Wireless Communications and Mobile Computing, Vol. 2020, 2020. 